\title{
Perfil das gestantes atendidas em um ambulatório no Rio Grande do Sul e o uso de substâncias psicoativas
}

\author{
Profile of pregnant women seen at an outpatient clinic in Rio Grande do Sul and the use of \\ psychoactive substances
}

Perfil de gestantes atendidas en un ambulatorio de Rio Grande do Sul y uso de sustancias psicoactivas

Amanda do Rosário Tavares ${ }^{1 *}$, Juliane Portella Ribeiro ${ }^{1}$, Adrize Rutz Porto ${ }^{1}$, Karen Barcelos Lopes ${ }^{1}$, Melissa Hartmann ${ }^{1}$, Eduarda Ramos de Leon ${ }^{1}$, Marina Soares Mota ${ }^{1}$.

\section{RESUMO}

Objetivo: Identificar o perfil sociodemográfico, gineco-obstétrico e uso de substâncias psicoativas de gestantes atendidas em um ambulatório de alto risco. Métodos: Estudo quantitativo, observacional, descritivo, com amostra de 431 gestantes. A análise dos dados foi descritiva por meio do Software SPSS. Resultados: A prevalência de idade foi entre 25 e 35 anos, solteiras, ensino médio completo e sem atividade laboral. Majoritariamente encontrava-se no terceiro trimestre gestacional, primigesta, sem filhos e sem aborto prévio. A substância psicoativa mais utilizada na vida foi o álcool $(81,0 \%)$, seguido do tabaco $(40,1 \%)$ e maconha $(12,8 \%)$. O tabaco foi a substância com maior uso nos últimos três meses, sendo consumido diariamente por $53(12,3 \%)$ gestantes, enquanto o álcool foi consumido por $68(15,8 \%)$ uma ou duas vezes. A maconha por sua vez foi usada com menor frequência nos últimos três meses. Conclusão: O predomínio de uso de substâncias lícitas (álcool e tabaco), tanto na vida como nos últimos três meses e a frequência de uso das mesmas, apontam um panorama desafiador para os profissionais de saúde com a necessidade de investimento em estratégias de educação em saúde; abordando os danos acarretados à saúde ao longo da vida, bem como suas consequências no período gravídico-puerperal.

Palavras-chave: Gravidez de alto risco, Usuários de drogas, Saúde materno-infantil, Cuidado pré-natal.

\begin{abstract}
Objective: To identify the sociodemographic and gynecological-obstetric profile and the use of psychoactive substances in pregnant women treated at a high-risk outpatient clinic. Methods: Quantitative, observational and descriptive study, with a sample of 431 pregnant women. Data analysis was descriptive using the SPSS Software. Results: The prevalence of age was between 25 and 35 years old, single, complete high school and without work activity. Mostly, they were in the third trimester of pregnancy, primigravidas, without children and without prior miscarriage. The psychoactive substance most used in life was alcohol ( $81.0 \%)$, followed by tobacco $(40.1 \%)$ and marijuana (12.8\%). Tobacco was the substance with the greatest use in the last three months, being consumed daily by $53(12.3 \%)$ pregnant women, while alcohol was consumed by $68(15.8 \%)$ once or twice. In turn, marijuana has been used less frequently in the past three months. Conclusion: The predominance of use of legal substances (alcohol and tobacco), both in life and in the last three months and the frequency of their use, point to a challenging scenario for health professionals, thus raising the need for investing in health education strategies; addressing the damage caused to health throughout life, as well as its consequences in the pregnancy-puerperal period.
\end{abstract}

Keywords: High-risk pregnancy, Drug users, Maternal and child health, Prenatal care.

1 Universidade Federal de Pelotas, Pelotas - RS. *E-mail: arosariotavares@icloud.com 


\section{RESUMEN}

Objetivo: Identificar el perfil sociodemográfico y ginecológico-obstétrico y el consumo de sustancias psicoactivas en mujeres embarazadas en un dispensario médico de alto riesgo. Métodos: Estudio cuantitativo, observacional y descriptivo, con una muestra de 431 embarazadas. El análisis de los datos fue descriptivo mediante el programa informático SPSS. Resultados: La prevalencia de edad fue entre 25 y 35 años, solteras, escuela secundaria completa y sin actividad laboral. En su mayoría, ellas estaban en el tercer trimestre de embarazo, eran primigestas, sin hijos y sin aborto previo. La sustancia psicoactiva más consumida en la vida fue el alcohol $(81,0 \%)$, seguida del tabaco $(40,1 \%)$ y la marihuana $(12,8 \%)$. El tabaco fue la sustancia de mayor consumo en los últimos tres meses, siendo consumida diariamente por $53(12,3 \%)$ mujeres embarazadas, mientras que el alcohol fue consumido por $68(15,8 \%)$ una o dos veces. A su vez, la marihuana se ha consumido con menos frecuencia en los últimos tres meses. Conclusión: El predominio del uso de sustancias legales (alcohol y tabaco), tanto en la vida como en los últimos tres meses y la frecuencia de su consumo, apuntan a un escenario desafiante para los profesionales de la salud, suscitando la necesidad de invertir en estrategias de educación en salud; abordando los daños ocasionados a la salud a lo largo de la vida, así como sus consecuencias en el período gestacional-puerperal.

Palabras clave: Embarazo de alto riesgo, Drogadictos, Salud maternal e infantil, Cuidado prenatal.

\section{INTRODUÇÃO}

A gestação é um evento fisiológico, de grandes transformações na vida da mulher e de sua família, com um desfecho favorável na maior parte dos casos. Existem algumas intercorrências gestacionais ocasionadas por problemas e/ou agravos, que passam a caracterizar a gestação em alto risco. A gestação de alto risco é composta por uma série de fatores clínicas, obstétricas e/ou sociais que se não observadas durante o período gestacional poderão ocasionar um desfecho negativo para o binômio mãe-feto (BRASIL, 2016; RODRIGUES ARM, et al., 2017).

Estima-se que cerca de $10 \%$ das gestações são classificadas como alto risco. Dentre os fatores classificatórios, destaca-se o uso, em qualquer quantidade, de substâncias psicoativas (SPA) tanto as lícitas quanto as ilícitas, cuja prevalência em gestantes do estado do Maranhão foi de 27,99\% (BRASIL, 2008; BRASIL, 2012; ROCHA PC et al., 2016) superando a Síndrome Hipertensiva da Gravidez (SHG) e o Diabetes Mellitus Gestacional (DMG) (BRASIL, 2012; BRASIL, 2016).

Em casos de gestação de alto risco, o acesso a outras redes assistenciais, como as de média e alta complexidade deverá ser garantido através do sistema de referência e contra referência, conforme a organização da linha de cuidado materno- infantil (BRASIL, 2012). É de suma importância quando falamos em prevenção de agravos na gestação citar a capacitação precoce, visto que este é o primeiro dos dez passos para um pré-natal de qualidade na Atenção Básica (AB), tendo como um de seus objetivos o desenvolvimento gestacional positivo (BRASIL, 2004; BRASIL, 2016).

Visando ofertar um cuidado integral e de qualidade para a gestante bem como a diminuição da taxa de mortalidade materna, torna-se necessário conhecer a singularidade desta mulher e suas características demográficas, socioeconômica, obstétricas e reprodutivas. Com isso é possível à identificação precoce dos fatores de risco, entre eles os problemas gestacionais e o uso de substâncias psicoativas, dado que gestantes expostas às substâncias apresentam incidências superiores de complicações clínicas e obstétricas (LEITE VC, et al., 2019; ANTUNES MC, et al., 2018).

O etanol consumido pela gestante consegue atravessar a barreira placentária permitindo que o feto seja exposto às mesmas concentrações de álcool que o sangue materno, contudo sua exposição torna-se maior devido ao metabolismo e excreção mais lenta. As crianças expostas ao álcool durante o período fetal apresentam uma maior probabilidade de desenvolver transtornos mentais, relacionado ao uso de substâncias psicoativas, e comportamentais como a personalidade antissocial e hiperatividade, tanto na adolescência e como na vida adulta (SANTOS RMS e GAVIOLI A, 2017; ROCHA PC, 2016; MAIA JA, et al., 2015; SANTOS RS, et al., 2017). 
Já, uso de substâncias ilícitas gera um impacto maior ainda na vida da gestante e do bebê, uma vez que as anfetaminas e cocaína são transportadas junto com os transportadores de nutrientes, permitindo que haja a competição favorável, reduzindo a absorção dos nutrientes pelo feto causando déficit de crescimento, malformações congênitas, edema agudo de pulmão, icterícia e sofrimento fetal (FERREIRA BRM e MIRANDA JKS, 2018).

Mesmo sendo preconizado, o acompanhamento de gestantes usuárias de álcool e outras drogas na Atenção Primária à Saúde (APS) e o encaminhamento de casos de maior gravidade para Centro de Atenção Psicossocial (CAPS), observa-se que essas gestantes se mantêm distantes dos serviços de saúde e do acompanhamento pré-natal como consequência do preconceito sofrido. Quando optam por realizá-lo, grande parte não relata o uso de substâncias psicoativas (BRASIL, 2012; ANTUNES MB, et al., 2018) o que dificulta tanto o vínculo, o cuidado e o seguimento adequado.

Investigar o perfil sociodemográfico e gineco-obstétrico desponta como uma possibilidade de criação de novas estratégias de cuidado condizentes às necessidades das gestantes usuárias de substâncias psicoativas (VECCHIA SLD, 2018). Desta forma, o objetivo do presente estudo é identificar o perfil sociodemográfico, gineco-obstétrico e uso de substâncias psicoativas de gestantes atendidas em um ambulatório de alto risco e tem como questão norteadora: "Qual o perfil sociodemográfico, gineco-obstétrico e uso de substâncias psicoativas de gestantes atendidas em um ambulatório de alto risco?"

\section{MÉTODOS}

Estudo quantitativo do tipo observacional, descritivo, em que os dados são coletados e analisados através de variáveis, a generalização e objetivação dos resultados através de uma amostra que aponta consequências a uma população (ESPERÓN JMT, 2017). Os dados utilizados nesse estudo é um recorte de uma pesquisa maior intitulada "Uso de Substâncias Psicoativas por gestantes de alto risco e puérperas atendidas no Ambulatório do Hospital Escola (HE)/Universidade Federal de Pelotas (UFPel)/Empresa Brasileira de Serviços Hospitalares (EBSERH)", estes por sua vez foram coletados no Ambulatório do HE/UFPel/EBSERH, localizado no município de Pelotas/RS, com atendimentos 100\% SUS. O Ambulatório de Especialidades é referência para a realização do pré-natal de alto risco, através de consultas com médicos especialistas desde o período gestacional até o retorno pós-parto (EBSERH, 2017).

A pesquisa supracitada foi submetida ao Comitê de Ética em Pesquisa na Área da Saúde, sendo aprovada pelo parecer № 2.843.605 e Certificado de Apresentação para Apreciação Ética (CAAE) № 96034518.6.0000.5316. Respeitando a Resolução 466/12 do Conselho Nacional de Saúde do Ministério da Saúde, que trata sobre a pesquisa envolvendo seres humanos e o Código de Ética dos Profissionais de Enfermagem embasado na Resolução Conselho Federal de Enfermagem (COFEN) 311/2017 Cap. III, Art. 89, 90 e 91 que trata das responsabilidades e deveres e os Art. 94, 96 e 98 que se refere às proibições (COFEN, 2012). A fim de assegurar o anonimato, as participantes da pesquisa foram identificadas por um número ordinal, correspondente a sequência em que foi realizada da coleta de dados.

A amostra foi calculada a partir da população estimada de 493 gestante de alto risco, empregando erro amostral $2 \%$, nível de confiança de $95 \%$ e considerando a prevalência de $28 \%$ de gestantes usuárias de substâncias psicoativas, licitas e ilícitas, encontrada no estudo de Rocha PC, et al. (2016) seria necessária uma amostra de 394 gestantes e puérperas. Foram acrescidos $10 \%$ considerando possíveis perdas ou recusas, assim a amostra consiste em 431 gestantes entrevistadas no período de agosto de 2018 a julho de 2019.

A coleta de dados foi feita através de dois instrumentos. Primeiramente foi aplicado um questionário semiestruturado elaborado pela pesquisadora do projeto como uma parte do instrumento de pesquisa para conhecer o perfil sociodemográfico e gineco-obstétrico da amostra. Subsequente a isto, foi aplicado o teste Alcohol, Smoking and Substance Involvement Screening Test (ASSIST), produzido pela Organização Mundial da Saúde (OMS), surge com a finalidade de complementar o Alcohol Use Disorders Identification Test (AUDIT), visto que o AUDIT avalia somente o uso de álcool, e o uso nocivo de substâncias psicoativas representa um grave problema de saúde pública. Tem como propósito auxiliar na triagem do uso problemático de substâncias psicoativas (FORMIGONI MLOS, et al., 2017). 
O ASSIST é composto por oito questões que exploram a frequência de uso de substâncias lícitas e ilícitas na vida e nos últimos três meses, problemas relacionados ao uso, preocupação referente ao uso por parte de pessoas próximas ao usuário, prejuízo e/ou dificuldade na execução de tarefas cotidianas, tentativas falhas de cessar ou reduzir o uso, sentimento de compulsão e uso de drogas injetáveis. Seu principal objetivo é a captação de pessoas que utilizam alguma substância, sendo ela lícita ou ilícita (HENRIQUE IFS, 2004). Em situações que o participante nega ter feito uso alguma vez na vida o questionário é encerrado. Nesse estudo serão utilizadas as questões 1 que investiga o uso de substâncias lícitas e ilícitas alguma vez na vida e a questão 2 que investiga a frequência do uso destas substâncias nos últimos três meses.

Para a análise dos dados, foi utilizada a estatística descritiva por meio de frequência absoluta e relativa, através do Software estatístico Statistical Package for the Social Sciences (SPSS) v. 22.0.

\section{RESULTADOS}

A idade mínima das gestantes foi de 13 anos e máxima de 46, com destaque entre 25 à 35 anos ( $n=223$; $51,9 \%)$, com mais de oito anos de estudo, solteira e sem atividade laboral (Tabela 1).

Tabela 1 - Perfil sociodemográfico das gestantes atendidas em um ambulatório referência em pré- natal de alto risco no período de agosto de 2018 a julho de 2019 - Pelotas, RS. $n=431$

\begin{tabular}{cc}
\hline Variáveis & $\mathbf{n}(\%)$ \\
\hline Faixa Etária (em anos) $^{\star}$ & \\
\hline$<15$ & $4(0,9)$ \\
15 a 24 & $126(29,3)$ \\
25 a 35 & $223(51,9)$ \\
$>35$ & $77(17,9)$ \\
\hline Estado Civil $^{\star}$ & \\
\hline Solteira & $232(54,0)$ \\
Casada/União Estável & $195(45,3)$ \\
Divorciada & $2(0,5)$ \\
Viúva & $1(0,2)$ \\
\hline Escolaridade $^{*}$ & \\
\hline Ensino Fundamental Incompleto & $94(21,8)$ \\
Ensino Fundamental Completo & $50(11,6)$ \\
Ensino Médio Incompleto & $70(16,2)$ \\
Ensino Médio Completo & $143(33,1)$ \\
Ensino Superior Incompleto & $36(8,4)$ \\
Ensino Superior Completo & $30(7,0)$ \\
Pós-Graduação & $8(1,9)$ \\
\hline Atividade Laboral & \\
\hline Não & $230(54,0)$ \\
Sim & $196(46,0)$ \\
\hline
\end{tabular}

Legenda: ${ }^{\star} \mathrm{n}<431$ por conta de dados omissos.

Fonte: Tavares AR, et al., 2020.

Quanto às informações gineco-obstétricas e clínicas (Tabela 2), a média da idade gestacional encontrada foi de $24,87( \pm 6,99)$ semanas, variando de sete a 41 semanas. As gestantes eram predominantemente primigestas e nulíparas, sendo a média de filhos de 1,28 $( \pm 1,33)$.

As condições clínicas mais frequentes foram 74 gestantes relataram Diabetes Mellitus Gestacional (DMG) ( $n=74 ; 17,2 \%)$, Doença Hipertensiva Específica da Gestação (DHEG) $(n=53 ; 12,3 \%)$, DHEG associada com DMG $(n=37 ; 8,6 \%)$, gestação gemelar $(n=15 ; 3,5 \%)$, hipo/hipertireoidismo $(n=10 ; 2,3 \%)$, Infeção do Trato Urinário (ITU) ( $n=9 ; 2,1 \%)$. Foram mencionadas também idade materna avançada $(n=7 ; 1,6 \%)$, aborto anterior $(n=7 ; 1,6 \%)$, hemorragias $(n=7 ; 1,6 \%)$, toxoplasmose $(n=6 ; 1,4 \%)$. As demais gestantes $(n=104 ; 24,1 \%)$ apontaram outro tipo de problema relacionado a atual gestação. 
Tabela 2 - Dados Gineco-obstétrico e Clínico das gestantes atendidas em um ambulatório referência em prénatal de alto risco no período de agosto de 2018 a julho de 2019 - Pelotas, RS. $n=431$.

\begin{tabular}{|c|c|}
\hline Variáveis & $\mathbf{N}(\%)$ \\
\hline \multicolumn{2}{|c|}{ 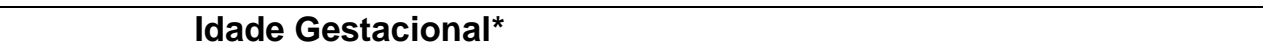 } \\
\hline $1^{\circ}$ trimestre & $55(12,9)$ \\
\hline $2^{\circ}$ trimestre & $168(39,4)$ \\
\hline 3 o trimestre & $203(47,7)$ \\
\hline \multicolumn{2}{|c|}{ № de Gestações* } \\
\hline 1 & $131(30,5)$ \\
\hline 2 & $111(25,9)$ \\
\hline 3 & $85(19,8)$ \\
\hline 4 ou mais & $102(23,8)$ \\
\hline \multicolumn{2}{|l|}{ № de Abortos* } \\
\hline Zero & $297(69,4)$ \\
\hline 1 a 2 & $123(28,8)$ \\
\hline 3 a 4 & $8(1,8)$ \\
\hline \multicolumn{2}{|l|}{ № de filhos* } \\
\hline Zero & $146(34,2)$ \\
\hline 1 & $123(28,8)$ \\
\hline 2 & $94(22,0)$ \\
\hline 3 & $39(9,1)$ \\
\hline 4 & $17(4,0)$ \\
\hline 5 ou mais & $8(1,9)$ \\
\hline \multicolumn{2}{|c|}{ Internação hospitalar na gestação* } \\
\hline Não & $358(84,2)$ \\
\hline Sim & $67(15,8)$ \\
\hline \multicolumn{2}{|c|}{ Atendimento em outro serviço de saúde* } \\
\hline Não & $159(47,0)$ \\
\hline Sim & $179(53,0)$ \\
\hline \multicolumn{2}{|c|}{ Condições clínicas na gestação atual } \\
\hline Não/Não sei & $68(15,8)$ \\
\hline $\operatorname{Sim}$ & $363(84,2)$ \\
\hline \multicolumn{2}{|c|}{ Acompanhamento Psicológico* } \\
\hline Não & $403(94,2)$ \\
\hline Sim & $25(5,8)$ \\
\hline
\end{tabular}

Legenda: ${ }^{*}<431$ por conta de dados omissos.

Fonte: Tavares AR, et al., 2020.

Entre as gestantes entrevistadas, 70(16,2\%) referiram não ter usado nenhuma substância psicoativa e 180 $(41,8 \%)$ o uso de pelo menos uma substância ao longo da vida; podendo ser durante a gestação ou não. Em relação à quantidade de tipos de substâncias utilizadas pelas gestantes em algum momento da vida, 126 $(29,2 \%)$ consumiram dois tipos, $37(8,6 \%)$ três substâncias e $18(4,2 \%)$ referiram uso de quatro ou mais. Na Tabela 3, são apresentados os tipos de substâncias mais consumidas pelas gestantes entrevistadas. 
Tabela 3 - Substâncias utilizadas em algum momento da vida - Pelotas, RS. $n=431$.

\begin{tabular}{ccc}
\hline & Uso de SPA na vida & \\
\hline Variável & $\mathbf{n}(\%)$ & $\mathbf{n}(\%)$ \\
\hline Tipos de Substâncias & Não & Sim \\
\hline Derivados do tabaco & $258(59,9)$ & $173(40,1)$ \\
Álcool & $82(19,0)$ & $349(81,0)$ \\
Maconha & $376(87,2)$ & $55(12,8)$ \\
Cocaína/Crack & $412(95,6)$ & $19(4,4)$ \\
Anfetamina/Ecstasy & $424(98,4)$ & $7(1,6)$ \\
Inalantes & $427(99,1)$ & $4(0,9)$ \\
Hipnóticos/Sedativos & $417(96,8)$ & $14(3,2)$ \\
Alucinógenos & $426(98,8)$ & $5(1,2)$ \\
Opioides & $429(99,5)$ & $2(0,5)$ \\
\hline
\end{tabular}

Legenda: **Uso de opioides sem prescrição médica.

Fonte: Tavares AR, et al., 2020.

Na Tabela 4 pode-se visualizar o uso de substâncias utilizadas nos últimos três meses pelas gestantes; sendo que anfetaminas/ecstasy, inalantes, alucinógenos e opioides não constam na mesma, pois nenhuma entrevistada relatou seu uso recentemente. Nos últimos três meses, $286(66,5 \%)$ gestantes referiram não ter utilizado nenhuma substância. Já $115(26,7 \%)$ relataram utilizar pelo menos uma substância e, apenas 29 $(6,7 \%)$, referiram uso de dois ou mais tipos de substâncias.

Tabela 4 - Frequência de uso de SPA pelas gestantes pelo tipo de substância nos últimos três meses Pelotas, RS. $n=431$.

\begin{tabular}{cccccc}
\hline \multicolumn{7}{c}{ Uso de SPA nos últimos três meses } \\
\hline Variáveis & Nunca & 1 ou 2 vezes & Mensal & Semanal & Diariamente \\
\cline { 2 - 6 } & $\mathbf{n ~ ( \% )}$ & $\mathbf{n ~ ( \% )}$ & $\mathbf{n ~ ( \% )}$ & $\mathbf{n ~ ( \% )}$ & $\mathbf{n}(\%)$ \\
\hline Tipo de substâncias & & & & & \\
\hline Derivados do tabaco & $359(83,3)$ & $8(1,9)$ & $3(0,7)$ & $8(1,9)$ & $53(12,3)$ \\
Álcool & $336(78,0)$ & $68(15,8)$ & $15(3,5)$ & $11(2,6)$ & $1(0,2)$ \\
Maconha & $424(98,4)$ & $3(0,7)$ & - & $2(0,5)$ & $2(0,5)$ \\
Cocaína/Crack & $429(99,5)$ & - & - & - & $2(0,5)$ \\
Hipnóticos/Sedativos & $430(99,8)$ & - & - & - & $1(0,2)$ \\
\hline
\end{tabular}

Fonte: Tavares AR, et al., 2020.

\section{DISCUSSÃO}

A gravidez na adolescência é considerada um problema de saúde pública a nível mundial, visto que gera uma sobrecarga de necessidades fisiológica, psicológica e sociais que podem comprometer 0 desenvolvimento, principalmente materno. Mãe e feto podem apresentar risco para o desenvolvimento de doenças hemorrágicas como abortamentos e placenta prévia, ITU, anemia, hipertensão, parto prematuro, malformações congênitas e infecções pós-parto (DUARTE ES, et al., 2018; ARAÚJO RLD, et al., 2016).

A gestação tardia também é considerada de alto risco, uma vez que o binômio mãe-feto tem possibilidade de um desfecho perinatal desfavorável, devido à senilidade ovariana e condições clínicas pré-existentes que tendem a surgir com o aumento da idade (ALVES NCC, et al., 2017). Algumas das consequências são prematuridade, baixo peso ao nascer, pré-eclâmpsia, DMG e índice de Apgar baixo. Além disso, podem-se citar alterações patológicas que dificultam o trabalho de parto, aumentam o risco de abortamento, hemorragias e doenças genéticas (GUIMARÃES VA, et al., 2018). 
No que diz respeito ao grau de escolaridade, uma pesquisa realizada com 1.886 gestantes de alto risco nas cidades de Campinas, Porto Alegre e São Paulo no período de março a setembro de 2016, apontou que $205(50,7 \%)$ haviam concluído o Ensino Médio (FERNANDES JA, et al., 2019). O grau de escolaridade materno é tido como um fator de risco, pois está diretamente relacionado à adesão às consultas de pré-natal e mortalidade infantil (POMINI MC, et al., 2018; LEITE VC, et al., 2019). No presente estudo, tanto as gestantes com alto grau como as com baixo grau de escolaridade apresentaram número de consultas compatíveis ao recomendado pelo MS para a idade gestacional.

No presente estudo, das 431 gestantes de alto risco, 358 (85,2\%) não necessitou de internação hospitalar. Tal achado vai ao encontro dos resultados obtidos no estudo de coorte com 55.404 gestantes que realizariam o parto em hospitais financiados somente pelo SUS, no ano de 2012. O mesmo evidenciou que 8.199 $(14,8 \%)$ das gestantes faziam parte do grupo de alto risco e, da totalidade, 53.044 (95,7\%) não precisaram de internações na gestação por complicações obstétricas (MOURA BLA, et al., 2018).

São consideradas condições clínicas as doenças infectocontagiosas vividas durante a gestação atual (ITU de repetição, doenças do trato respiratório, toxoplasmose, rubéola, entre outras), além de doenças clínicas diagnosticadas pela primeira vez durante o período gestacional, isoimunização $R h$ anti-D (RhD) - Rh negativo, amniorrexe prematura (abaixo de 37 semanas), sangramentos de origem uterina e malformações fetais (MELO WA, et al., 2016).

Alusivo às consultas de pré-natal, o presente estudo aponta que grande parte das gestantes $(n=174$; $67,4 \%$ ) estava no terceiro trimestre e tinham quatro ou mais consultas realizadas. De acordo com a Portaria no 570 de $1^{\circ}$ de junho de 2000 do Ministério da Saúde é preconizado o início do pré-natal até o quarto mês gestacional, ou seja, até as 12 primeiras semanas e, que ao final da gestação, a parturiente tenha no mínimo seis consultas de pré-natal, sendo, preferencialmente, uma no primeiro trimestre, duas no segundo e três no terceiro trimestre da gestação (BRASIL, 2000). Assim, constata-se que as gestantes do presente estão em conformidade ao preconizado pelo Ministério da Saúde.

Considerando a gestação de alto risco, cabe ressaltar que a utilização de drogas ilícitas é um problema de saúde pública significativo, mas que ainda requer estudos epidemiológicos, principalmente no Brasil. Quando se citam gestantes usuárias de drogas, torna-se mais difícil observar o seu desfecho, uma vez que as gestantes usuárias são mais discriminadas, o que leva à negação do vício e não adesão pela assistência prénatal ou acesso tardio (ANDRADE CA, 2018; ROCHA PF, et al., 2016; WRONSKI JA, et al., 2016).

Estudo realizado em Recife também utilizando o ASSIST, no período de outubro de 2017 a dezembro de 2018, com uma amostra de 138 gestantes mostrou que dentre as drogas lícitas, a mais consumida em algum momento da vida foi o álcool $(n=59 ; 78,67 \%)$, seguido do tabaco $(n=23 ; 30,67 \%)$ (ARRIBAS CGS, et al., 2018). No que se referem às drogas ilícitas, neste mesmo estudo, a de maior uso foi a maconha $(n=12 ; 16 \%)$, posteriormente os inalantes $(n=10 ; 13,33 \%)$, anfetamina $(n=9 ; 12 \%)$, alucinógenos $(n=4 ; 5,33 \%)$, cocaína/crack ( $n=2 ; 2,67 \%)$ e opioides $(n=1 ; 1,33 \%)$. Quanto aos sedativos, $17(27,67 \%)$ gestantes relataram uso (ARRIBAS CGS, et al., 2018).

Os resultados acima apresentam poucas divergências do presente estudo, visto que a segunda substância ilícita mais consumida no estudo foi a cocaína/crack $(n=19 ; 4,4 \%)$ seguido de hipnóticos/sedativos $(n=14$; $3,2 \%)$, anfetaminas/ecstasy ( $n=7 ; 1,6 \%)$, alucinógenos $(n=5 ; 1,2 \%)$ e por fim, inalantes $(n=4 ; 0,9 \%)$. Santos RMS e Gavioli A (2017) também abordaram em seu estudo, anteriormente já citado, que das 209 gestantes, $179(86,1 \%)$ já tinha utilizado álcool alguma vez na vida, 73 (35,4\%) tabaco, $11(5,7 \%)$ maconha e duas $(1,4 \%)$ cocaína.

Em relação ao uso de substâncias e intercorrências gestacionais, das $115(63,1 \%)$ gestantes que fizeram uso de um tipo de substância nos últimos três meses, $91(79,1 \%)$ relataram ter alguma condição clínica associada a atual gestação e $24(20,9 \%)$ relataram não ter ou saber sobre as condições clínicas. De forma semelhante, estudo realizado em São Paulo, no período de agosto de 2015 a agosto de 2016, com uma amostra de 287 gestantes, mostrou que $86(29,9 \%)$ faziam uso de drogas, sendo elas lícitas e/ou ilícitas. Destas 86, 17 (30\%) tiveram aborto prévio, 21 (38\%) tinham transtornos mentais comuns e sete (13\%) tiveram alguma intercorrência durante o período gestacional (KASSADA DS, 2017). 
Uma pesquisa realizada na cidade de Santa Maria no Estado do Rio Grande do Sul (RS), com uma amostra de 255 gestantes atendidas nas UBS de Estratégia de Saúde da Família (ESF), 32 (12,55\%) relataram utilizar tabaco durante a gestação, sendo que $73,07 \%$ das mulheres ter o hábito de fumar no dia-a-dia. Referente ao álcool, $36(14,12 \%)$ eram consumidoras, porém grande parte das mulheres $(69,70 \%)$ relataram raramente beber durante a gestação (BIANCHINI BV, 2019).

Os problemas relacionados à ingesta de álcool e o uso substâncias psicoativas, como maconha, crack e cocaína tem revelado um importante aumento entre as mulheres, principalmente no período gravídicopuerperal, incluindo admissão em hospitais públicos como resultado de intercorrências (clínicas, cirúrgicas e obstétricas) (RENNER F, et al., 2016).

Pesquisa realizada no Centro de Controle de Intoxicação (CCl) em Maringá/PR, no período de 2008 a 2015, com uma amostra de 57 gestantes, observou que a faixa etária das gestantes usuárias de substâncias psicoativas eram dos 20 aos 24 anos $(38,6 \%)$ e a droga mais utilizada foi o crack $(77,2 \%)$, seguido do tabaco $(49,1 \%)$, maconha $(40,3 \%)$, álcool $(19,3 \%)$ e cocaína (15,8\%). Destas 57 gestantes, $27(47,4 \%)$ tiveram internações hospitalares devido à intercorrências clínicas/obstétricas $(47,4 \%)$, parto prematuro $(42,1 \%)$, causas externas/violências (8,8\%) e puerpério imediato (1,7\%) (MARANGONI SR, et al., 2017).

O rastreamento do uso de substâncias psicoativas durante o período gestacional é algo raro e que não está inserido no cotidiano de grande parte dos profissionais devido aos estigmas impostos, desconhecimento quanto à prevalência e recursos terapêuticos. Além disso, gestantes usuárias de substâncias sentem-se inseguras para relatar tal uso com seus cuidadores por conta de julgamentos, principalmente por parte dos profissionais da saúde (KANADA SBFM, 2019).

O enfermeiro, como integrante da equipe de saúde e sendo o coordenador da equipe de enfermagem, está apto a realizar o acolhimento destas gestantes usuárias de substâncias psicoativas, e deve preparar sua equipe de enfermagem, bem como os agentes comunitários de saúde para realizar a melhor abordagem, visando à promoção da assistência à saúde e à redução de danos (FONESECA PMM, et al., 2017).

Como limitação, o presente estudo aponta a impossibilidade de generalização dos dados obtidos, visto que o mesmo foi desenvolvido em um serviço específico que atende uma população com características próprias, no entanto os resultados encontrados são semelhantes às diversas literaturas pesquisadas.

\section{CONCLUSÃO}

Os resultados do presente estudo mostraram a importância de conhecer o perfil sociodemográfico, ginecoobstétrico, bem como o uso de substâncias psicoativas e sua frequência, já que grande parte das gestantes usuárias de substâncias estão inseridas em um contexto de vulnerabilidade social e necessitam de cuidados de qualidade ofertados pelos profissionais de saúde. O predomínio de uso de substâncias lícitas (álcool e tabaco), tanto na vida como nos últimos 3 meses e a frequência de uso das mesmas, apontam um panorama desafiador para os profissionais de saúde com a necessidade de investimento em estratégias de educação em saúde na atenção primária; abordando os danos acarretados à saúde ao longo da vida, bem como suas consequências para o binômio mãe e feto no período gravídico-puerperal. Conhecer a frequência e modo como são utilizadas as substâncias psicoativas pelas gestantes é de suma importância, uma vez que tendo acesso a esta informação e conhecendo o perfil destas mulheres é possível realizar uma intervenção adequada e eficaz, em muitos casos estimulando a abstinência total da droga e protegendo o binômio mãe e feto de desfechos perinatais desfavoráveis.

\section{AGRADECIMENTOS E FINANCIAMENTO}

O artigo está vinculado ao projeto de pesquisa "Uso de substâncias psicoativas por gestantes de alto risco e puérperas atendidas no ambulatório do HE/UFPEL/EBSERH". O presente estudo teve como fonte de financiamento a Fundação de Amparo à Pesquisa do Estado do Rio Grande do Sul (FAPERGS) com a bolsa Iniciação Cientifica - Edital CPESQ № 003/2019. 


\section{REFERÊNCIAS}

1. ALVES NCC, et al. Complicações na gestação em mulheres com idade maior ou igual a 35 anos. Revista Gaúcha de Enfermagem, Porto Alegre, 2017, 38 (4): e2017-0042.

2. ANDRADE CA de. Uso de álcool e drogas durante a gestação: resultado materno e perinatal. Dissertação (Mestrado) - Faculdade Montes Belos, Goiás, 2018; 71p.

3. ANTUNES MBD, et al. Desfecho perinatal em gestantes usuárias de drogas atendidas em um centro especializado. Revista Eletrônica Saúde Mental Álcool Drogas, Ribeirão Preto, 2018, 14 (4): 211-218.

4. ARAÚJO RLM, de et al. Gravidez na adolescência: consequências centralizadas para a mulher. Temas Saúde [online], 2016, 16 (2): 567-587.

5. ARRIBAS CGSM, de et al. Positividade ao consumo de álcool e outras drogas por mulheres gestantes em três hospitais públicos do recife a partir da aplicação do teste ASSIST. Em: Anais do Congresso Brasileiro de Saúde Coletiva. Campinas: Galoá. 2018.

6. BIANCHINI BV. Prevalência de gestantes usuárias de drogas lícitas e suas percepções em relação ao pré-natal: um estudo misto. Dissertação (Mestrado Profissional em Saúde Materno Infantil) - Universidade Franciscana, Santa Maria - RS, 2019; 100p.

7. BRASIL. Ministério da Saúde. Secretaria de Atenção à Saúde. Departamento de Ações Programáticas Estratégicas. Gestação de alto risco: manual técnico / Ministério da Saúde, Secretaria de Atenção à Saúde, Departamento de Acões Programáticas Estratégicas. - 5. ed. - Brasília : Editora do Ministério da Saúde, 302p., 2012.

8. PREVENÇAO AO USO INDEVIDO DE DROGAS: Curso de Capacitação para Conselheiros Municipais. Brasília: Presidência da República, Secretaria Nacional Antidrogas - SENAD, 2008. 288 p.

9. MINISTÉRIO DA SAÚDE. Federação Brasileira das Associações de Ginecologia e Obstetrícia. Sociedade Brasileira de Diabetes. Rastreamento e diagnóstico de diabetes mellitus gestacional no Brasil. Brasília, DF: OPAS, 32p., 2016.

10. MINISTÉRIO DA SAÚDE. Portaria no 570 de 10 de junho de 2000 . Brasília, 2000.

11. MINISTÉRIO DA SAÚDE. Protocolos da Atenção Básica: Saúde das Mulheres / Ministério da Saúde, Instituto SírioLibanês de Ensino e Pesquisa - Brasília: Ministério da Saúde, 203p. 2016.

12. COFEN. Conselho Federal de Enfermagem. Resolução COFen ํo 466 de 12 de dezembro de 2012. Trata de pesquisas e testes em seres humanos. Brasília, DF: CỎFEN, 2012.

13. DUARTE ES, et al. A gravidez na adolescência e suas consequências Biopsicossociais. Revista De Ciência em Foco, 2018, 2 (1): 45-52.

14. EBSERH. Empresa Brasileira de Serviços Hospitalares. Hospital Escola da Universidade Federal de Pelotas. Carta de Serviços ao Cidadão, 2017.

15. ESPERON JMT. Pesquisa Quantitativa na Ciência da Enfermagem. Escola Anna Nery, Rio de Janeiro, 2017,21 (1): e20170027.

16. FERNANDES JÁ, et al. Perfil das gestantes de alto risco e a cogestão da decisão sobre a via de parto entre médico e gestante. Saúde em debate., Rio de Janeiro, 2019, 43 (121): 406-16.

17. FERREIRA BRM, MIRANDA JKS. As complicações causadas pelo consumo de drogas lícitas e ilícitas durante a gestação: um desafio para a equipe de enfermagem. Revista Científica de Enfermagem, 2018, 6 (18): 36-43.

18. FONSECA PMM, et al. Gestante usuária de crack: desafios encontrados no pré-natal. Revista Científica UMC, 2017, $2(2)$.

19. FORMIGONI MLOS, et al. ASSIST - Eixo de Instrumentos. Aberta, 2017.

20. GUIMARÃES VA, et al. Prevalência e fatores associados ao uso de álcool durante a gestação em uma maternidade de Goiás, Brasil Central. Ciência e saúde coletiva, Rio de Janeiro, 2018. 23 (10): 3413-3420.

21. HENRIQUEE IFS, et al. Validação da versão brasileira do teste de triagem do envolvimento com álcool, cigarro e outras substâncias (ASSIST). Revista da Associação Médica do Brasil, São Paulo, 2004, 50 (2): 199-206.

22. KANADA SBFM. Linha de cuidado como dispositivo para a adesão de gestantes usuárias de drogas aos cuidados do pré-natal. Dissertação (Programa de Estudos Pós-Graduados em Educação nas Profissões da Saúde) - Pontifícia Universidade Católica de São Paulo, Sorocaba, 2019; 106 p.

23. KASSADA DS. Gestantes na atenção primária à saúde: transtornos mentais comuns, qualidade de vida e uso de drogas. Tese (Doutorado em Enfermagem Psiquiátrica) - Escola de Enfermagem de Ribeirão Preto, Universidade de São Paulo, Ribeirão Preto, 2017.

24. LEITE VC, et al. Estratificação de risco em gestantes no pré- natal. Revista Uningá, [S.I.], 2019,56 (S2): 184-193.

25. MAIA JA, et al. Consequências do uso de drogas durante a gravidez. Revista de Enfermagem Contemporânea, 2015, 4 (2): 121-128.

26. MARANGONI SR, GAVIOLI A. Perfil sociodemográfico das mulheres usuárias de álcool e outras drogas na gravidez. Revista Uningá Review, 2017, 30 (3): 19-24.

27. MELO WA, et al. Gestação de alto risco: fatores associados em município do Noroeste paranaense. Espaç saúde [Online], 2016, 17 (1): 83-92.

28. MOURA BLA, et al. Internações por complicações obstétricas na gestação e desfechos maternos e perinatais, em uma coorte de gestantes no Sistema Único de Saúde no Município de São Paulo, Brasil. Caderno de Saúde Pública, Rio de Janeiro, 2018, 34 (1): e00188016.

29. POMINI MC et al. Conhecimento de gestantes sobre o teste da linguinha em neonatos. Revista de odontologia da UNESP, Araraquara, 2018, 47 (6): 341-347.

30. RENNER $F$, et al. Avaliação do uso de drogas por gestantes em um hospital universitário do interior do Rio Grande do Sul. Jornal de Epidemiologia e Controle de Infecção [Internet]. 2016, 6 (2).

31. ROCHA PC, et al. Prevalência e fatores associados ao uso de drogas ilícitas em gestantes da coorte BRISA. Caderno de Saúde Pública, 2016, 32(1): e00192714.

32. RODRIGUES ARM, et al. Gravidez de alto risco: Análise dos determinantes de saúde. SANARE, 2017, 16 (1): $23-28$.

33. SANTOS RS, et al. Prevenção da síndrome alcoólica fetal: subsídios para a prática de enfermeiras obstétricas. Revista Enfermagem UERJ, 2017, 25: e27793.

34. SANTOS RMS e GAVIOLI A. Risco relacionado ao consumo de drogas de abuso em gestantes. Revista Rene, 2017, $18(1): 35-42$.

35. VECCHIA SLD. Perfil epidemiológico das gestantes encaminhadas ao pré-natal de alto risco. Trabalho de Conclusão de Curso - Universidade Federal da Fronteira Sul, 2018; 129p.

36. WRONSKI JL, et al. Uso do crack na gestação: vivências de mulheres usuárias. Revista de Enfermagem UFPE [online], 2016, 10 (4): 1231-1239. 